\title{
Lapatinib/Letrozole Regimen
}

National Cancer Institute

\section{Source}

National Cancer Institute. Lapatinib/Letrozole Regimen. NCI Thesaurus. Code C142860.

A chemotherapy regimen consisting of lapatinib and letrozole that is used as a treatment for advanced hormone receptor (HR)-positive, HER-2/neu-positive breast cancer. 